\title{
VECTOR-VALUED MODULAR FORMS FROM THE MUMFORD FORMS, SCHOTTKY-IGUSA FORM, PRODUCT OF THETANULLWERTE AND THE AMAZING KLEIN FORMULA
}

\author{
MARCO MATONE AND ROBERTO VOLPATO
}

(Communicated by Kathrin Bringmann)

\begin{abstract}
Vector-valued Siegel modular forms are the natural generalization of the classical elliptic modular forms as seen by studying the cohomology of the universal abelian variety. We show that for $g \geq 4$, a new class of vectorvalued modular forms, defined on the Teichmüller space, naturally appears from the Mumford forms, a question directly related to the Schottky problem. In this framework we show that the discriminant of the quadric associated to the complex curves of genus 4 is proportional to the square root of the products of Thetanullwerte $\chi_{68}$, which is a proof of the recently rediscovered Klein "amazing formula". Furthermore, it turns out that the coefficients of such a quadric are derivatives of the Schottky-Igusa form evaluated at the Jacobian locus, implying new theta relations involving the latter, $\chi_{68}$ and the theta series corresponding to the even unimodular lattices $E_{8} \oplus E_{8}$ and $D_{16}^{+}$. We also find, for $g=4$, a functional relation between the singular component of the theta divisor and the Riemann period matrix.
\end{abstract}

\section{INTRODUCTION}

Here we briefly describe the results and introduce some simple (linear) algebraic facts that will be useful in this paper and describe our notation for theta functions.

For each fixed positive integers $g, n$, define

$$
M_{n}:=\left(\begin{array}{c}
g+n-1 \\
n
\end{array}\right), N_{n}:=(2 n-1)(g-1)+\delta_{n 1}, K_{n}:=M_{n}-N_{n},
$$

so that, for a complex curve $C$ of genus $g \geq 2, M_{n}$ and $N_{n}$ are the dimensions of $\operatorname{Sym}^{n} H^{0}\left(K_{C}\right)$ and $H^{0}\left(K_{C}^{n}\right)$, respectively. Let

$$
\mathfrak{H}_{g}:=\left\{\left.Z \in M_{g}(\mathbb{C})\right|^{t} Z=Z, \operatorname{Im} Z>0\right\}
$$

be the Siegel upper half-space, i.e. the space of $g \times g$ complex symmetric matrices with positive definite imaginary part, and define the usual action of the symplectic group $\Gamma_{g}:=\operatorname{Sp}(2 g, \mathbb{Z})$ on $\mathfrak{H}_{g}$ by $Z \mapsto(A Z+B)(C Z+D)^{-1},\left(\begin{array}{ll}A & B \\ C & D\end{array}\right) \in \Gamma_{g}$. Denote by $\mathcal{M}_{g}$ the moduli stack of complex curves of genus $g$ and by $\mathcal{A}_{g}=\Gamma_{g} \backslash \mathfrak{H}_{g}$ the moduli stack of principally polarized abelian $g$-folds. According to Torelli's Theorem the morphism Jac : $\mathcal{M}_{g} \rightarrow \mathcal{A}_{g}$, which on points takes the algebraic curves to its Jacobian, is injective. The question of characterizing the image of Jac is the Schottky problem.

Received by the editors June 26, 2011 and, in revised form, October 27, 2011.

2010 Mathematics Subject Classification. Primary 14H42; Secondary 14H40, 14H55. 
Let $\mathcal{C}_{g} \stackrel{\pi}{\longrightarrow} \mathcal{M}_{g}$ be the universal curve over $\mathcal{M}_{g}$ and $L_{n}=R \pi_{*}\left(K_{\mathcal{C}_{g} / \mathcal{M}_{g}}^{n}\right)$ the vector bundle on $\mathcal{M}_{g}$ of rank $N_{n}$ with fiber $H^{0}\left(K_{C}^{n}\right)$ at the point of $\mathcal{M}_{g}$ representing $C$. On $\mathcal{A}_{g}$ it is the conormal bundle to the zero section in the universal abelian scheme; it pulls back via Jac to $\mathcal{M}_{g}$. A vector-valued Siegel modular form on $\mathcal{A}_{g}$ is a global section of some tensor bundle of the Hodge vector bundle on $\mathcal{A}_{g}$, whose pull-back to $\mathcal{M}_{g}$ via the Torelli map corresponds to $L_{1}$. In spite of their relevance, e.g. in number theory, they have been studied essentially only in the case of genus two, where they correspond to suitable commutators of Siegel modular forms. In Section 2 we will introduce a new set of vector-valued modular forms, defined on the Teichmüller space and strictly related to the Mumford forms, which are holomorphic global sections of the bundle

$$
\mathbb{F}_{g}=\left(\operatorname{det} \mathbb{E}_{g}\right)^{c_{n}} \otimes\left(\bigwedge^{N_{n}} \operatorname{Sym}^{n} \mathbb{E}_{g}\right)^{*}
$$

on $\mathcal{M}_{g}$, where $\mathbb{E}_{g} \equiv L_{1}$ and $c_{n}:=6 n^{2}-6 n+1$. Their weight is $c_{n}-\left(\begin{array}{c}g+n-1 \\ n-1\end{array}\right)$.

Such vector-valued Teichmüller modular forms provide a deep relation between Schottky's problem, quadrics describing $C$ and $\theta$-functions. The construction fits into the general problem of finding explicit formulae that relate theta nulls, which can be regarded as homogeneous coordinates on the moduli space of abelian varieties (with appropriate level structure), to projective invariants of curves, which are homogeneous coordinates on $\mathcal{M}_{g}$. Such formulae are also very interesting for physics, since they appear naturally in the computation of higher loop superstring amplitudes [1]-[5]. As a first application of the vector-valued Teichmüller modular forms, in Section 3 we consider the case $g=4$. In particular, we will show that the discriminant of the quadric associated to $C$ is proportional to the square root of the products of Thetanullwerte $\chi_{68}$, which is a proof of the recently rediscovered Klein "amazing formula". The relation with the Schottky's problem is due to the fact that the coefficients of such a quadric are derivatives with respect to the period matrix of the Schottky-Igusa form. This provides new theta relations involving the latter, $\chi_{68}$ and the theta series corresponding to the even unimodular lattices $E_{8} \oplus E_{8}$ and $D_{16}^{+}$. Finally, we will find a functional relation between the singular component $\Theta_{s}$ of the theta divisor and the Riemann period matrix and show that the hyperelliptic locus is a zero of order 10 for the Hessian of $\theta(e, Z), e \in \Theta_{s}$.

Given a basis $v_{1}, \ldots, v_{g}$ of a $g$-dimensional vector space $V$, denote by $\tilde{v}_{1}^{(n)}, \ldots, \tilde{v}_{M_{n}}^{(n)}$ the basis of the symmetrized tensor product $\operatorname{Sym}^{n} V$ given by elements of the form

$$
\frac{1}{n !} \sum_{\pi \in S_{n}} v_{\pi\left(i_{1}\right)} \otimes \cdots \otimes v_{\pi\left(i_{n}\right)},
$$

with $S_{n}$ the group of permutations of $n$ objects, taken with respect to an arbitrary ordering.

Proposition 1.1. Let $V \cong \mathbb{C}^{g}$ be a g-dimensional complex vector space and fix $A \in G L(V)$. Then, the induced endomorphism on the 1-dimensional space $\bigwedge^{M_{n}}\left(\operatorname{Sym}^{n} V\right) \cong \mathbb{C}$ is given by $\operatorname{det} A^{\left(\begin{array}{c}g+n-1 \\ n-1\end{array}\right)} \in G L_{1}(\mathbb{C})$. Explicitly, if $\tilde{w}_{i}=$ $\sum_{j=1}^{g} A_{i j} \tilde{u}_{j}, u \in V$, then

$$
\tilde{w}_{1}^{(n)} \wedge \cdots \wedge \tilde{w}_{M_{n}}^{(n)}=\operatorname{det} A^{\frac{n}{g} M_{n}} \tilde{u}_{1}^{(n)} \wedge \cdots \wedge \tilde{u}_{M_{n}}^{(n)} .
$$


Consider the theta function with characteristics

$$
\theta\left[\begin{array}{l}
a \\
b
\end{array}\right](z, Z):=\sum_{k \in \mathbb{Z}^{g}} e^{\pi i^{t}(k+a) Z(k+a)+2 \pi i^{t}(k+a)(z+b)},
$$

where $z \in \mathbb{C}^{g}, Z \in \mathfrak{H}_{g}$ and $a, b \in \mathbb{R}^{g}$. Let $\left\{\alpha_{1}, \ldots, \alpha_{g}, \beta_{1}, \ldots, \beta_{g}\right\}$ be a symplectic basis of $H_{1}(C, \mathbb{Z})$. Denote by $\left\{\omega_{i}\right\}_{1 \leq i \leq g}$ the basis of $H^{0}\left(K_{C}\right)$ satisfying the normalization condition $\oint_{\alpha_{i}} \omega_{j}=\delta_{i j}$, and by $\tau_{i j}:=\oint_{\beta_{i}} \omega_{j} \in \mathfrak{H}_{g}$ the Riemann period matrix, $i, j=1, \ldots, g$. The basis of $H_{1}(C, \mathbb{Z})$ is determined up to the transformation

$$
\left(\begin{array}{c}
\alpha \\
\beta
\end{array}\right) \mapsto\left(\begin{array}{c}
\tilde{\alpha} \\
\tilde{\beta}
\end{array}\right)=\left(\begin{array}{cc}
D & C \\
B & A
\end{array}\right)\left(\begin{array}{c}
\alpha \\
\beta
\end{array}\right), \quad \gamma \equiv\left(\begin{array}{cc}
A & B \\
C & D
\end{array}\right) \in \Gamma_{g},
$$

which induces the following transformation on the period matrix:

$$
\tau \mapsto \gamma \cdot \tau=(A \tau+B)(C \tau+D)^{-1} .
$$

For $n \in \mathbb{Z}$, denote by $J_{n}(C)$ the principal homogeneous space of linear equivalence classes of divisors of degree $n$ on $C$. The Jacobian $J(C):=\mathbb{C}^{g} / L_{\tau}, L_{\tau}:=$ $\mathbb{Z}^{g}+\tau \mathbb{Z}^{g}$, is identified with $J_{0}(C)$ : each point of $J_{0}(C)$ can be expressed as $D_{2}-D_{1}$, with $D_{1}$ and $D_{2}$ effective divisors of the same degree, which corresponds to $\int_{D_{1}}^{D_{2}} \omega \in J(C)$. Choose an arbitrary point $p_{0} \in C$ and let $A(p):=$ $\left(A_{1}(p), \ldots, A_{g}(p)\right), A_{i}(p):=\int_{p_{0}}^{p} \omega_{i}, p \in C$, be the Abel-Jacobi map. It embeds $C$ into the Jacobian $J_{0}(C)$ and generalizes to a map from the space of divisors of $C$ into $J_{0}(C)$ as $A\left(\sum_{i} n_{i} p_{i}\right):=\sum_{i} n_{i} A\left(p_{i}\right), p_{i} \in C, n_{i} \in \mathbb{Z}$. By the Jacobi Inversion Theorem the restriction of $A$ to the space $C_{g}$ of divisors of degree $g$ on $C$ is a surjective map onto $J_{0}(C)$. Consider the vector of Riemann constants $K_{i}^{p}:=\frac{1}{2}+\frac{1}{2} \tau_{i i}-\sum_{j \neq i}^{g} \oint_{\alpha_{j}} \omega_{j} \int_{p}^{x} \omega_{i}, i=1, \ldots, g$, for all $p \in C$. For any $p \in C$ define the formal sum $\Delta:=(g-1) p-K^{p}$ so that, for any divisor $\xi$ of degree $g-1$ in $C, \xi-\Delta$ is the point in $\mathbb{C}^{g}$ given by $\int_{(g-1) p}^{\xi} \omega+K^{P}$. Under the projection $\mathbb{C}^{g} \rightarrow J_{0}(C), \Delta$ becomes a distinguished point in $J_{g-1}(C)$ depending only on the homological class of the marking (recall that a marking for $C$ is given by fixing a canonical homotopy basis together with a basepoint $p_{0} \in C$; see e.g. 6]). Furthermore, $2 \Delta=K_{C}$. We refer to [7] and [6] for further details.

If $\delta^{\prime}, \delta^{\prime \prime} \in\{0,1 / 2\}^{g}$, then $\theta[\delta](z, \tau):=\theta\left[\begin{array}{l}\delta^{\prime} \\ \delta^{\prime \prime}\end{array}\right](z, \tau)$ has definite parity in $z$ $\theta[\delta](-z, \tau)=e(\delta) \theta[\delta](z, \tau)$, where $e(\delta):=e^{4 \pi i^{t} \delta^{\prime} \delta^{\prime \prime}}$. There are $2^{2 g}$ different characteristics of definite parity. By the Abel Theorem each one of such characteristics determines the divisor class of a spin bundle $L_{\delta} \simeq K_{C}^{\frac{1}{2}}$, so that we may call them spin structures. There are $2^{g-1}\left(2^{g}+1\right)$ even and $2^{g-1}\left(2^{g}-1\right)$ odd spin structures.

We will also consider the prime form $E(z, w)$ and the multi-valued $g / 2$-differential $\sigma(z)$ on $C$ with empty divisor, satisfying the property

$$
\sigma\left(z+{ }^{t} \alpha n+{ }^{t} \beta m\right)=\chi^{-g} e^{\pi i(g-1)^{t} m \tau m+2 \pi i^{t} m \mathcal{K}^{z}} \sigma(z) .
$$

Such conditions fix $\sigma(z)$ only up to a factor independent of $z$. The precise definition, to which we will refer, can be given, following [6], on the universal covering of $C$ (see also [7]). 
Proposition 1.2. For each integer $n$, let $\phi^{n}:=\left\{\phi_{i}^{n}\right\}_{1 \leq i \leq N_{n}}$ be an arbitrary basis of $H^{0}\left(K_{C}^{n}\right)$. Then

$$
\kappa\left[\phi^{1}\right]:=\frac{\operatorname{det} \phi_{i}^{1}\left(p_{j}\right) \sigma(y) \prod_{1}^{g} E\left(y, p_{i}\right)}{\theta\left(\sum_{1}^{g} p_{i}-y-\Delta\right) \prod_{1}^{g} \sigma\left(p_{i}\right) \prod_{i<j}^{g} E\left(p_{i}, p_{j}\right)},
$$

for all $p_{1}, \ldots, p_{g}, y \in C$, and

$$
\kappa\left[\phi^{n}\right]:=\frac{\operatorname{det} \phi_{i}^{n}\left(p_{j}\right)}{\theta\left(\sum_{1}^{N_{n}} p_{i}-(2 n-1) \Delta\right) \prod_{1}^{N_{n}} \sigma\left(p_{i}\right)^{2 n-1} \prod_{i<j}^{N_{n}} E\left(p_{i}, p_{j}\right)},
$$

for $n \geq 2$, for all $p_{1}, \ldots, p_{N_{n}} \in C$, depend only on the marking of $C$ and on $\left\{\phi_{i}^{n}\right\}_{1 \leq i \leq N_{n}}$.

Proof. For each integer $n, \kappa\left[\phi^{n}\right]$ is a meromorphic function on $C$ with empty divisor [7, 8.

\section{Vector-VAlued Teichmüller modular FORMS FROM THE MUMFORD FORM}

Let $\lambda_{n}:=\operatorname{det} L_{n}$ be the determinant line bundle. According to Mumford 9 ]

$$
\lambda_{n} \cong \lambda_{1}^{\otimes c_{n}}
$$

where $c_{n}:=6 n^{2}-6 n+1$. The Mumford form $\mu_{g, n}$ is the unique, up to a constant, holomorphic section of $\lambda_{n} \otimes \lambda_{1}^{-\otimes c_{n}}$ nowhere vanishing on $\mathcal{M}_{g}$.

Comparing $\mu_{g, 2}$ with the Polyakov measure for the bosonic string, Manin observed that $c_{2}=13$ in Mumford's formula coincides with half of the string critical dimension. In a seminal paper [10] Belavin and Knizhnik proved that the Polyakov measure coincides with $\left|\mu_{g, 2}\right|^{2}$. More generally $-c_{n}$ is the central charge of the chiral $b-c$ system of conformal weight $n$ [11].

Belavin and Knizhnik obtained $\mu_{g, 2}$ from an expression for the curvature form of the determinant of Laplace operators. As observed in [12, this is a particular case of the similar formula for the determinants of Dirac operators on arbitrary compact manifolds, due to Bismut and Freed 13 (see also 14 and the references therein). Such results lead to expressions in terms of complex geometry of the canonical curve $C$, providing a link with the spectral invariants which appear using the formulae for the Laplace operator determinants by Ray and Singer [15] and leading to sums over lengths of closed geodesics by means of the Selberg trace formula.

The expression of $\mu_{g, 2}$ in terms of $\theta$-functions has been derived in the context of string theory by Beilinson and Manin in [16.

Theorem 2.1. Let $\left\{\phi_{i}^{n}\right\}_{1 \leq i \leq N_{n}}$ be a basis of $H^{0}\left(K_{C}^{n}\right), n \geq 2$. The Mumford form is, up to a universal constant,

$$
\mu_{g, n}=\frac{\kappa[\omega]^{(2 n-1)^{2}}}{\kappa\left[\phi^{n}\right]} \frac{\phi_{1}^{n} \wedge \cdots \wedge \phi_{N_{n}}^{n}}{\left(\omega_{1} \wedge \cdots \wedge \omega_{g}\right)^{c_{n}}} .
$$

The expression of $\mu_{g, n}$ in terms of $\theta$-functions, given by Verlinde and Verlinde [17] and Fay [6], follows immediately by (1.6) and (1.7). Nevertheless, it remains the hard question of expressing $\mu_{g, n}$ without using points on $C$. As we will see, there are exceptions for $\mu_{2,2}$ and $\mu_{3,2}$. There is also a proof for the 
long-standing conjecture for $\mu_{4,2}$; here we show that it follows immediately in the present context. A related issue concerning $\kappa[\omega]^{(2 n-1)^{2}} / \kappa\left[\phi^{n}\right]$ is its dependence on the basis $\left\{\phi_{i}^{n}\right\}_{1 \leq i \leq N_{n}}$ : apparently, there is no natural choice for such a basis. In the case of $\mu_{2,2}$ and $\mu_{3,2}$, since $K_{2}=0$ (i.e., $\operatorname{Sym}^{2} H^{0}\left(K_{C}\right)$ and $H^{0}\left(K_{C}^{2}\right)$ have the same dimension), the natural choice is to use $\left\{\omega_{i} \omega_{j}\right\}_{1 \leq i, j \leq g}$ as a basis of $H^{0}\left(K_{C}^{2}\right)$. It turns out that such a choice reveals new interesting properties just as in the case when $K_{n}>0$. Actually, the application of symmetric products of the $\omega_{i}$ 's in this case introduces free vectorial indices and therefore leads to vector-valued modular forms defined on the Teichmüller space (see [18 for a very nice account of vector-valued Siegel modular forms), which are strictly related to the investigations in [8, 19, 20]. Remarkably, such a structure will also lead to a strict connection between Mumford forms, quadrics describing canonical curves, their discriminant and the Schottky problem. In particular, for $g=4$ we will get some new results connecting the above structures to the Schottky-Igusa form, the theta series and the products of Thetanullwerte $\chi_{68}$, where

$$
\chi_{k}(Z):=\prod_{\delta \text { even }} \theta[\delta](0, Z)
$$

$Z \in \mathfrak{H}_{g}$, with $k=2^{g-2}\left(2^{g}+1\right)$. Let $C$ be a Riemann surface of genus $g \geq 2$ with a given symplectic basis for $H_{1}(C, \mathbb{Z})$. For each positive integer $n$, consider the basis $\tilde{\omega}_{1}^{(n)}, \ldots, \tilde{\omega}_{M_{n}}^{(n)}$ of $\operatorname{Sym}^{n} H^{0}\left(K_{C}\right)$ whose elements, as in (1.1), are symmetrized tensor products of $n$-tuples of vectors of the basis $\omega_{1}, \ldots, \omega_{g}$, taken with respect to an arbitrary ordering chosen once and for all. Denote by $\omega_{i}^{(n)}, i=1, \ldots, M_{n}$, the image of $\tilde{\omega}_{i}^{(n)}$ under the natural map

$$
\psi: \operatorname{Sym}^{n} H^{0}\left(K_{C}\right) \rightarrow H^{0}\left(K_{C}^{n}\right)
$$

It is well known that such a map is surjective if and only if $g=2$ or $C$ is nonhyperelliptic of genus $g>2$. Of course, when $M_{n}=N_{n}$ the map is an isomorphism. In particular, for $n=2$, this is the case for $g=2$ and $g=3$ non-hyperelliptic. It has been shown in [21, 22, 23] that for $g=2, \frac{\kappa[\omega]^{9}}{\kappa\left[\omega^{(2)}\right]}=\frac{1}{\pi^{12} \chi_{5}^{2}(\tau)}$. Furthermore, it has been conjectured in [21, 22] and proved in [24, 26] that for $g=3, \frac{\kappa[\omega]^{9}}{\kappa\left[\omega^{(2)}\right]}=\frac{1}{2^{6} \pi^{18} \chi_{18}^{1 / 2}(\tau)}$.

Remark 2.2. Under (1.4) we have $\omega_{i} \mapsto \omega_{i}^{\prime}:=\sum_{j=1}^{g} \omega_{j}(C \tau+D)_{j i}^{-1}, i=1, \ldots, g$. Such a transformation property induces the $\Gamma_{g}$-actions $\tilde{\rho}^{(n)}$ on $\operatorname{Sym}^{n} H^{0}\left(K_{C}\right)$ and $\rho^{(n)}:=\psi \circ \tilde{\rho}^{(n)}$ on $H^{0}\left(K_{C}^{n}\right)$. Explicitly,

$$
\rho^{(n)}(\gamma) \cdot\left(\omega_{i_{1}} \cdots \omega_{i_{n}}\right)=\sum_{j_{1}, \ldots, j_{n}=1}^{g} \omega_{j_{1}} \cdots \omega_{j_{n}}(C \tau+D)_{j_{1} i_{1}}^{-1} \cdots(C \tau+D)_{j_{n} i_{n}}^{-1}
$$

$\gamma \equiv\left(\begin{array}{l}A B \\ C D\end{array}\right) \in \Gamma_{g}, i_{1}, \ldots, i_{n}=1, \ldots, g$. Furthermore, by (1.2),

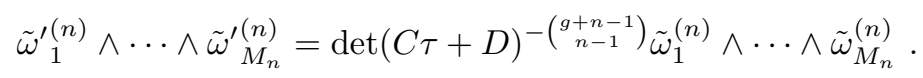


Definition 2.3. Let $n \geq 2$ be an integer. For each $i_{1}, \ldots, i_{K_{n}} \in\left\{1, \ldots, M_{n}\right\}$ and for all $x_{1}, \ldots, x_{N_{n}} \in C$, define $\left[i_{1}, \ldots, i_{K_{n}} \mid \tau\right]$ to be completely antisymmetric in $i_{1}, \ldots, i_{K_{n}}$ and such that, for any permutation $\pi$ of $M_{n}$ objects,

$$
\begin{aligned}
{\left[\pi\left(N_{n}+1\right), \ldots, \pi\left(M_{n}\right) \mid \tau\right] } & \operatorname{sgn}(\pi) \operatorname{det}_{1 \leq i, j \leq N_{n}} \omega_{\pi(i)}^{(n)}\left(x_{j}\right) \\
& :=\frac{{ }^{N_{n}} \sigma\left(x_{j}\right)^{2 n-1} \prod_{j<k}^{N_{n}} E\left(x_{j}, x_{k}\right)}{\kappa[\omega]^{(2 n-1)^{2}} \theta\left(\sum_{1}^{N_{n}} x_{j}-(2 n-1) \Delta\right) \prod_{1}^{N^{2}}} .
\end{aligned}
$$

$\left[i_{1}, \ldots, i_{K_{n}} \mid \tau\right]$ is independent of $\pi$ and of $x_{1}, \ldots, x_{N_{n}} \in C$. In fact, analogously to Proposition 1.2. one can check that it is a meromorphic function with zero divisor with respect to each $x_{i}$ (see [6, 7]). This definition allows us to express the generators of the kernel of the map $\psi$ in $(2.2)$ in terms of the basis $\tilde{\omega}_{1}^{(n)}, \ldots, \tilde{\omega}_{M_{n}}^{(n)}$ in a very simple form.

Proposition 2.4. For each integer $n \geq 2$ and for all $i_{2}, \ldots, i_{K_{n}} \in\left\{1, \ldots, M_{n}\right\}$ we have

$$
\sum_{i=1}^{M_{n}}\left[i, i_{2}, \ldots, i_{K_{n}} \mid \tau\right] \omega_{i}^{(n)}(x)=0 .
$$

Proof. If $i_{2}, \ldots, i_{K_{n}}$ are not pairwise distinct, this is obvious. Otherwise, the lefthand side of (2.5) is proportional to

$$
\sum_{\pi}\left[\pi\left(N_{n}+1\right), \ldots, \pi\left(M_{n}\right) \mid \tau\right] \omega_{\pi\left(N_{n}+1\right)}^{(n)}(x),
$$

where the sum is over the $\pi$ in $S_{M_{n}}$ such that $\pi\left(N_{n}+2\right)=i_{2}, \ldots, \pi\left(M_{n}\right)=i_{K_{n}}$. Thus, (2.5) is equivalent to $\operatorname{det}_{\substack{i \in J \leq N_{n}+1 \\ 1 \leq j \leq N^{\prime}}} \omega_{i}^{(n)}\left(x_{j}\right)=0$, with $J=\left\{1, \ldots, M_{n}\right\}$ । $\left\{i_{2}, \ldots, i_{K_{n}}\right\}$ and $x_{N_{n}+1} \equiv x$.

If we identify a non-hyperelliptic smooth $C$ with its canonical model in $\mathbb{P} H^{0}\left(K_{C}\right)$, then the relations (2.5) generate the ideal of hypersurfaces of degree $n$ containing the canonical curve.

Let $\mathcal{H}_{g}$ be the closure of the locus of hyperelliptic Riemann period matrices in $\mathfrak{H}_{g}$. Consider the basis

$$
\eta_{j}=z^{j-1} d z / w,
$$

$j=1, \ldots, g$ of $H^{0}\left(K_{C}\right)$, with $C$ the hyperelliptic curve $w^{2}=\prod_{j=1}^{2 g+2}\left(z-z_{j}\right)$.

Proposition 2.5. $\left[i_{N_{n}+1}, \ldots, i_{M_{n}} \mid \tau\right]$ are holomorphic, not identically vanishing global sections of the bundle

$$
\mathbb{F}_{g}=\left(\operatorname{det} \mathbb{E}_{g}\right)^{c_{n}} \otimes\left(\bigwedge^{N_{n}} \operatorname{Sym}^{n} \mathbb{E}_{g}\right)^{*} \cong\left(\operatorname{det} \mathbb{E}_{g}\right)^{d_{n}} \otimes\left(\bigwedge^{K_{n}} \operatorname{Sym}^{n} \mathbb{E}_{g}^{*}\right)
$$

on $\mathcal{M}_{g}$, where

$$
d_{n}:=6 n^{2}-6 n+1-\left(\begin{array}{c}
g+n-1 \\
n-1
\end{array}\right)
$$

vanishing precisely when $\omega_{i_{1}}^{(n)}, \ldots, \omega_{i_{N_{n}}}^{(n)}$ is not a basis of $H^{0}\left(K_{C}^{n}\right)$. In particular, they have zeroes of order at least $(n-1)(g-1)-1$ at $\tau \in \mathcal{H}_{g}$. 
Proof. Comparing Definition 2.3 with (1.7) and (2.1) yields

$$
\left[i_{N_{n}+1}, \ldots, i_{M_{n}} \mid \tau\right]=\frac{\epsilon_{i_{1}, \ldots, i_{M_{n}}} \omega_{i_{1}}^{(n)} \wedge \cdots \wedge \omega_{i_{N_{n}}}^{(n)}}{\left(\omega_{1} \wedge \cdots \wedge \omega_{g}\right)^{c_{n}} \mu_{g, n}}
$$

$i_{1}, \ldots, i_{M_{n}} \in\left\{1, \ldots, M_{n}\right\}$, where $\epsilon_{i_{1}, \ldots, i_{M_{n}}}$ is the completely antisymmetric tensor with $\epsilon_{1, \ldots, M_{n}}=1$. Holomorphicity follows by the fact that $\mu_{g, n}$ is holomorphic on $\mathcal{M}_{g}$, and since it is nowhere vanishing on $\mathcal{M}_{g}$, even its inverse is holomorphic on $\mathcal{M}_{g}$. In particular, (2.8) shows that $\left[i_{N_{n}+1}, \ldots, i_{M_{n}} \mid \tau\right]$ and $\omega_{i_{1}}^{(n)} \wedge \cdots \wedge \omega_{i_{N_{n}}}^{(n)}$ have the same divisor so that $\left[i_{N_{n}+1}, \ldots, i_{M_{n}} \mid \tau\right]$ does not vanish identically on $\mathcal{M}_{g}$. Furthermore, since $\eta_{g}^{n}=z^{n(g-1)}(d z)^{n} / w^{n}$, it follows that on the hyperelliptic loci $\operatorname{Sym}^{n} H^{0}\left(K_{C}\right)$ is a $n(g-1)+1$-dimensional subspace of $H^{0}\left(K_{C}^{n}\right)$ so that, by (2.8), $\left[i_{1}, \ldots, i_{K_{n}} \mid \tau\right]$ vanishes at order at least $(n-1)(g-1)-1$ at $\tau \in \mathcal{H}_{g}$. The modular properties of $\epsilon_{i_{1}, \ldots, i_{M_{n}}} \omega_{i_{1}}^{(n)} \wedge \cdots \wedge \omega_{i_{N_{n}}}^{(n)}$ are the same as $\epsilon_{i_{1}, \ldots, i_{M_{n}}} \tilde{\omega}_{i_{1}}^{(n)} \wedge \cdots \wedge \tilde{\omega}_{i_{N_{n}}}^{(n)}$, which, in turn, can be derived explicitly considering the identity

$\tilde{\omega}_{1}^{(n)} \wedge \cdots \wedge \tilde{\omega}_{M_{n}}^{(n)}=\sum_{i_{N+1}, \ldots, i_{M_{n}}=1}^{M_{n}} \epsilon_{i_{1}, \ldots, i_{M_{n}}}\left(\tilde{\omega}_{i_{1}}^{(n)} \wedge \cdots \wedge \tilde{\omega}_{i_{N_{n}}}^{(n)}\right) \otimes \tilde{\omega}_{i_{N_{n}+1}}^{(n)} \otimes \cdots \otimes \tilde{\omega}_{i_{M_{n}}}^{(n)}$.

Noting that under (1.4)

$$
\frac{\tilde{\omega}_{1}^{\prime(n)} \wedge \cdots \wedge \tilde{\omega}_{M_{n}}^{(n)}}{\left(\omega_{1}^{\prime} \wedge \cdots \wedge \omega_{g}^{\prime}\right)^{c_{n}} \mu_{g, n}}=\operatorname{det}(C \tau+D)^{d_{n}} \frac{\tilde{\omega}_{1}^{(n)} \wedge \cdots \wedge \tilde{\omega}_{M_{n}}^{(n)}}{\left(\omega_{1} \wedge \cdots \wedge \omega_{g}\right)^{c_{n}} \mu_{g, n}}
$$

we obtain

$$
\begin{aligned}
\sum_{i_{N+1}, \ldots, i_{M_{n}}=1}^{M_{n}} & {\left[i_{N_{n}+1}, \ldots, i_{M_{n}} \mid \gamma \cdot \tau\right] \tilde{\omega}_{i_{N_{n+1}}^{\prime}}^{(n)} \otimes \cdots \otimes \tilde{\omega}_{i_{M_{n}}}^{(n)} } \\
& =\operatorname{det}(C \tau+D)^{d_{n}} \sum_{k_{N+1}, \ldots, k_{M_{n}}=1}^{M_{n}}\left[k_{N_{n}+1}, \ldots, k_{M_{n}} \mid \tau\right] \tilde{\omega}_{k_{N_{n+1}}}^{(n)} \otimes \cdots \otimes \tilde{\omega}_{k_{M_{n}}}^{(n)},
\end{aligned}
$$

where $\gamma \equiv\left(\begin{array}{l}A \\ C D\end{array}\right) \in \Gamma_{g}, \gamma \cdot \tau$ given in (1.5). It follows that, under (1.4),

$$
\begin{aligned}
& \sum_{i_{1}, \ldots, i_{K_{n}}=1}^{M_{n}} \rho^{(n)}(\gamma)_{k_{1} i_{1}} \cdots \rho^{(n)}(\gamma)_{k_{K_{n}} i_{K_{n}}} {\left[i_{1}, \ldots, i_{K_{n}} \mid \gamma \cdot \tau\right] } \\
&=\operatorname{det}(C \tau+D)^{d_{n}}\left[k_{1}, \ldots, k_{K_{n}} \mid \tau\right]
\end{aligned}
$$

so that $\left[i_{1}, \ldots, i_{K_{n}} \mid \tau\right]$ defines a section of the vector bundle

$$
\left(\operatorname{det} \mathbb{E}_{g}\right)^{d_{n}} \otimes\left(\bigwedge^{K_{n}} \operatorname{Sym}^{n} \mathbb{E}_{g}^{*}\right) \cong\left(\operatorname{det} \mathbb{E}_{g}\right)^{c_{n}} \otimes\left(\bigwedge^{N_{n}} \operatorname{Sym}^{n} \mathbb{E}_{g}\right)^{*}
$$

Here we used the isomorphisms

$$
\left(\bigwedge^{N_{n}} V\right)^{*} \cong \bigwedge^{M_{n}} V^{*} \otimes \bigwedge^{K_{n}} V^{*}
$$

and

$$
\bigwedge^{M_{n}} \operatorname{Sym}^{n} W^{*} \cong(\operatorname{det} W)^{-\left(\begin{array}{c}
g+n-1 \\
n-1
\end{array}\right)}
$$


that hold for a generic complex vector bundle $V$ and $W$ of rank $M_{n}$ and $g$, respectively (see also Proposition 1.1).

\section{Discriminant of THE $g=4$ QUADRIC AND THE SCHOTTKY-IGUSA MODULAR FORM}

Denote by $\mathcal{I}_{g}$ the closure of the locus of Riemann period matrices in $\mathfrak{H}_{g}$. The elements of such a locus can be naturally identified with the elements of the Torelli space $\mathcal{T}_{g}$. The Torelli space is the quotient of the Teichmüller space by the Torelli group, that is, the normal subgroup of the mapping class group whose elements act trivially on $H_{1}(C, \mathbb{Z})$. Equivalently, the Torelli group is the kernel of the natural homomorphism of the mapping class group into the symplectic group $S p(2 g, \mathbb{Z})$. Note that the hyperelliptic sublocus $\mathcal{H}_{g} \subset \mathfrak{H}_{g}$ can be naturally identified with the sublocus of $\mathcal{T}_{g}$ corresponding to hyperelliptic Riemann surfaces. We denote by $\hat{\mathcal{T}}_{g}$ its complement in the Torelli space, whose elements correspond to non-hyperelliptic surfaces.

The explicit expression of the coefficients of a quadric containing a canonical curve of genus $g$ obviously depends on the choice of a coordinate basis of $\mathbb{P}^{g-1}$ or, equivalently, of a basis of $H^{0}\left(K_{C}\right)$. Therefore, it is natural to look for quantities characterizing such a curve that are invariant under the projective linear group $\mathbb{P G L}(g, \mathbb{C})$ of coordinate changes on $\mathbb{P}^{g-1}$. We denote by $I_{k}$ an invariant of weight $k$, i.e. a function of the coefficients $C_{i j}$ of the quadric, transforming as

$$
I_{k}(C)=\operatorname{det} \rho(A)^{k} I_{k}(A \cdot C),
$$

where $A$ is an element of $\operatorname{GL}(g, \mathbb{C}), \rho: \operatorname{GL}(g, \mathbb{C}) \rightarrow \operatorname{End}\left(\mathbb{C}^{g}\right)$ is the fundamental representation and $A \cdot C$ denotes the action of $A \in \mathrm{GL}(g, \mathbb{C})$ on the coefficients $C_{i j}$.

If a symplectic basis of $H_{1}(C, \mathbb{Z})$ is fixed, such an invariant can be evaluated with respect to the basis of holomorphic abelian differentials canonically normalized with respect to the $\alpha$-periods. It follows, by definition, that $I_{k}$ must transform as

$$
I_{k} \rightarrow I_{k}^{\prime}=\operatorname{det}(C \tau+D)^{k} I_{k}
$$

under a $\Gamma_{g}$-transformation corresponding to a change of the symplectic basis of $H_{1}(C, \mathbb{Z})$.

For $g=4$ and $n=2$, Proposition 2.4 gives the quadric

$$
\sum_{i, j=1}^{4} \frac{1+\delta_{i j}}{2}[(i j) \mid \tau] \omega_{i} \omega_{j}=0 .
$$

Here, $(i j)$ denotes the element in $\left\{1, \ldots, M_{2}(4)=10\right\}$ such that $\omega_{(i j)}^{(2)}=\omega_{i} \omega_{j}$ in the chosen ordering for $\omega_{1}^{(2)}, \ldots, \omega_{10}^{(2)}$.

Define

$$
\Delta_{4}(\tau):=\operatorname{det}_{i j}\left(\frac{1+\delta_{i j}}{2}[(i j) \mid \tau]\right) .
$$

For all $i, j=1, \ldots, 4$ and $Z \in \mathfrak{H}_{4}$, set

$$
S_{4 i j}(Z):=\frac{1+\delta_{i j}}{2} \frac{\partial F_{4}(Z)}{\partial Z_{i j}}
$$

where

$$
F_{g}(Z)=2^{g} \sum_{\delta \text { even }} \theta^{16}[\delta](0, Z)-\left(\sum_{\delta \text { even }} \theta^{8}[\delta](0, Z)\right)^{2},
$$


$Z \in \mathfrak{H}_{g}$, is a modular form of weight 8 . Up to normalization, it can be shown that $F_{g}$ is the difference of the theta series of the even unimodular lattices $E_{8} \oplus E_{8}$ and $D_{16}^{+}$:

$$
F_{g}(Z)=2^{2 g}\left(\Theta_{D_{16}^{+}}(Z)-\Theta_{E_{8}}^{2}(Z)\right) .
$$

$F_{4}$ is the Schottky-Igusa form [27, 28], and the irreducible variety in $\mathfrak{H}_{4}$ defined by $F_{4}=0$ is $\mathcal{I}_{4} \subset \mathfrak{H}_{4}$.

For each $\tau \in \mathcal{I}_{4}$, let $\Theta_{s}$ be the singular locus of the theta function, and set

$$
\sigma_{i j}(e, \tau):=\left.\frac{1+\delta_{i j}}{2} \frac{\partial \theta(e, Z)}{\partial Z_{i j}}\right|_{Z=\tau} .
$$

Proposition 3.1. $\Delta_{4}(\tau)$ is a Teichmüller modular form of weight 34 .

Proof. By (2.9), in the case $n=2$ and $g=4$,

$$
[(i j) \mid \gamma \cdot \tau]=\operatorname{det}(C \tau+D)^{8 t}(C \tau+D)[(k l) \mid \tau](C \tau+D)
$$

so that $\Delta_{4}(\gamma \cdot \tau)=\operatorname{det}(C \tau+D)^{34} \Delta_{4}(\tau)$.

Lemma 3.2. Let $C \in \mathcal{T}_{4}$ be a marked Riemann surface and let $\tau$ be its period matrix. Then

$$
\frac{1+\delta_{i j}}{2}[(i j) \mid \tau]=c S_{4 i j}(\tau)
$$

$i, j=1, \ldots, 4$, with $c \in \mathbb{C}^{\star}$ independent of $\tau$, so that

$$
\Delta_{4}(\tau)=c^{4} \operatorname{det} S_{4}(\tau) .
$$

Proof. Define some local coordinates $t_{1}, \ldots, t_{9}$ on $\mathcal{T}_{4}$ centered at the point $C$, corresponding to the period matrix $\tau \in \mathcal{I}_{4}$, and consider an arbitrary element $\partial_{t} \in T_{C} \mathcal{T}_{4}$ in the tangent space. Since $F_{4}$ vanishes identically on $\mathcal{I}_{4}$, we have

$$
0=\partial_{t} F_{4}(\tau)=\left.\sum_{i \leq j} \frac{\partial F_{4}}{\partial Z_{i j}}\right|_{Z=\tau} \partial_{t} \tau_{i j}=\left.\sum_{i \leq j} \frac{\partial F_{4}}{\partial Z_{i j}}\right|_{Z=\tau} d \tau_{i j}\left(\partial_{t}\right) .
$$

Here, $d \tau_{i j}$ is the element of the cotangent space $T_{C}^{*} \mathcal{T}_{4}$ defined by $d \tau_{i j}\left(\partial_{t}\right):=\partial_{t} \tau_{i j}$, for all $\partial_{t} \in T_{C} \mathcal{T}_{4}$. The Kodaira-Spencer map establishes an isomorphism between $T_{C}^{*} \mathcal{T}_{4}$ and $H^{0}\left(K_{C}^{2}\right)$. In particular, via Rauch's variational formula [6], $d \tau_{i j}$ corresponds to the quadratic differential $\omega_{i} \omega_{j}$. Since the identity (3.9) holds for an arbitrary $\partial_{t} \in T_{C} \mathcal{T}_{4}$, it follows that

$$
\left.\sum_{i \leq j} \frac{\partial F_{4}}{\partial Z_{i j}}\right|_{Z=\tau} \omega_{i} \omega_{j}=\sum_{i, j=1}^{4} S_{4 i j}(\tau) \omega_{i} \omega_{j}=0
$$

as an element in $H^{0}\left(K_{C}^{2}\right)$. Since the ideal of quadrics of a canonical curve is generated by (3.1), we have $S_{4 i j}(\tau)=c(\tau) \frac{1+\delta_{i j}}{2}[(i j) \mid \tau]$, for some holomorphic function $c(\tau)$ on $\mathcal{I}_{4}$, independent of $i, j=1, \ldots, 4$. Let us prove that $c(\tau)$ must be invariant under the action of $\Gamma_{4}$ on $\tau$. Since $F_{4}(\tau)=0$ for all $\tau \in \mathcal{I}_{4}$, it follows that on $\mathcal{I}_{4}$,

$$
S_{4}(\gamma \cdot \tau)=\operatorname{det}(C \tau+D)^{8 t}(C \tau+D) S_{4}(\tau)(C \tau+D),
$$

which is the same transformation property satisfied by $[(i j) \mid \tau]$. Thus, $c$ is modular invariant, so it must be a constant. Finally, observe that $c$ cannot vanish since it would imply $\frac{\partial F_{4}}{\partial Z_{i j}}(\tau)=0$ for all $\tau \in \mathcal{I}_{4}$, which is impossible because $F_{4}$ is irreducible [28]. 
It is now useful to recall the following well-known result.

Lemma 3.3. Let $C$ be either a non-hyperelliptic Riemann surface of genus $g=4$ or a non-trigonal surface of $g=5$. Then, the canonical model of $C$ is contained in a quadric of rank 3 if and only if $\prod_{\delta \text { even }} \theta[\delta]=0$.

Proof. The modular form $\prod_{\delta \text { even }} \theta[\delta]$ vanishes if and only if $C$ has an even singular spin structure $\delta$. In this case, there are two holomorphic sections $\xi_{1}$ and $\xi_{2}$ of $L_{\delta}$, $L_{\delta}^{2}=K_{C}$, so that taking $\eta_{1}=\xi_{1}^{2}, \eta_{2}=\xi_{2}^{2}$ and $\eta_{3}=\xi_{1} \xi_{2}$ one has $\eta_{3}^{2}=\eta_{1} \eta_{2}$, which is a quadric of rank 3 containing $C$.

Conversely, suppose that $\eta_{3}^{2}=\eta_{1} \eta_{2}$ for some $\eta_{1}, \eta_{2}, \eta_{3} \in H^{0}\left(K_{C}\right)$. Set $\left(\eta_{i}\right)=$ $\sum_{p \in C} m_{i}(p) p$ and consider the divisor $D=\sum_{p \in C} \min \left\{m_{1}(p), m_{3}(p)\right\} p . \quad D$ has degree at most $g-1$; otherwise the ratio $\eta_{1} / \eta_{3}$ would be a meromorphic function with at most $g-2$ poles and the curve would be hyperelliptic for $g=4$ or trigonal for $g=5$. On the other hand, $\eta_{3}^{2}=\eta_{1} \eta_{2}$ implies that $\left(\eta_{1}\right) \leq 2\left(\eta_{3}\right)$, and since the supports of $\left(\eta_{1}\right)-D$ and $\left(\eta_{3}\right)-D$ are disjoint, the only possibility is that $\left(\eta_{1}\right)=2 D$. Therefore, $\eta_{1}$ is the square of a holomorphic section of the line bundle $L_{\delta}$, with $L_{\delta}^{2}=K_{C}$, corresponding to the divisor $D$. By the same reasoning it follows that $\eta_{2}$ is the square of a holomorphic section of the line bundle $L_{\delta^{\prime}}$, with $L_{\delta^{\prime}}^{2}=K_{C}$, corresponding to the divisor $D^{\prime}$. Since $\left(\eta_{3}\right)=D+D^{\prime}$ is a canonical divisor it follows that $\delta^{\prime}=\delta$. Then $\delta$ necessarily corresponds to an even singular spin structure, since a surface of genus $g=4,5$ admits no odd super-singular spin structures, that is, spin structures with three or more holomorphic sections.

Theorem 3.4. For any $\tau \in \mathcal{I}_{4}$,

$$
\operatorname{det} S_{4}(\tau)=d \chi_{68}(\tau)^{1 / 2}
$$

where $d \in \mathbb{C}^{\star}$ is independent of $\tau$. In particular, by Lemma 3.2 ,

$$
\Delta_{4}(\tau)=\frac{d}{c^{4}} \chi_{68}(\tau)^{1 / 2}
$$

Furthermore,

$$
\chi_{68}(\tau)^{1 / 2}=\frac{2^{28}}{d} \operatorname{det}\left[\left.\left(1+\delta_{i j}\right) \frac{\partial}{\partial Z_{i j}}\left(\Theta_{D_{16}^{+}}-\Theta_{E_{8}}^{2}\right)\right|_{Z=\tau}\right] .
$$

Proof. By (3.10) it follows that $\operatorname{det} S_{4}(\gamma \cdot \tau)=\operatorname{det}(C \tau+D)^{34} \operatorname{det} S_{4}(\tau)$ so that $\operatorname{det} S_{4}$ is a modular form of weight 34 when restricted to $\mathcal{I}_{4}$. (Note that $\operatorname{det} S_{4}$ is not a modular form on the whole $\mathfrak{H}_{4}$, since the modular group action on $S_{4}$ is affine outside $\mathcal{I}_{4}$ ). On the other hand, it has been proved in [29] that the square root of $\chi_{68}$ on the right-hand side of (3.11) is well-defined when restricted to $\mathcal{I}_{4}$.

Choose $\tau \in \mathcal{I}_{4} \backslash \mathcal{H}_{4}$. By Lemma 3.2, the left-hand side of (3.11) is proportional to the discriminant. By Lemma 3.3, $\Delta_{4}(\tau)$ vanishes if and only if the Riemann surface has a singular even spin structure. The locus of Riemann surfaces with singular even spin structures in $\mathcal{T}_{4}$ corresponds to the divisor of $\sqrt{\prod_{\delta} \text { even } \theta[\delta](0, \tau)}$ in $\mathcal{I}_{4}$ [29] so that the meromorphic function

$$
\frac{\operatorname{det} S_{4}}{\sqrt{\prod_{\delta \text { even }} \theta[\delta](0, \tau)}}
$$

has no poles on $\mathcal{I}_{4} \backslash \mathcal{H}_{4}$ and therefore on $\mathcal{I}_{4}$, since $\mathcal{H}_{4}$ has codimension 2. Since it is a holomorphic modular invariant function not identically zero on $\mathcal{I}_{4}$, it must be a non-vanishing constant. Finally, (3.13) follows from (3.5) and (3.11). 
Theorem 3.5. Let $e \in \Theta_{s}$. The functional relation between $\Theta_{s}$ and $\tau \in \mathcal{I}_{4}$,

$$
S_{4 i j}(\tau)=\left(\frac{d \chi_{68}(\tau)^{1 / 2}}{\operatorname{det} \sigma(e, \tau)}\right)^{1 / 4} \sigma_{i j}(e, \tau),
$$

holds. Both $S_{4 i j}(\tau)$ and $\sigma_{i j}(e, \tau)$ have a zero of order at least 2 for all $\tau \in \mathcal{H}_{4}$ for any $i, j=1, \ldots, 4$. Furthermore, both $\operatorname{det} S_{4}$ and $\operatorname{det} \sigma(e, \tau)$ have a zero of order 10 for all $\tau \in \mathcal{H}_{4}$ and at least a zero when $e \in \Theta_{s}$ is an even $\theta$-characteristic for all $\tau \in \mathcal{I}_{4}$.

Proof. The well-known relation $\sum_{i, j=1}^{4} \sigma_{i j}(e, \tau) \omega_{i} \omega_{j}=0, e \in \Theta_{s}$, implies

$$
S_{4 i j}(\tau)=G(e \mid \tau) \sigma_{i j}(e, \tau),
$$

with $G(e \mid \tau)$ determined by Theorem 3.4 upon taking the determinant of both sides of (3.15). The fact that all the $\tau$ in $\mathcal{H}_{4}$ are zeros of order at least $(2-1)(4-1)-1=2$ of $S_{4 i j}(\tau)$ for any $i, j=1, \ldots, 4$ is an immediate consequence of Proposition 2.5 and Lemma 3.2. Concerning the order of the zero of $\operatorname{det} S_{4}$ and $\operatorname{det} \sigma(e, \tau)$ for all $\tau \in \mathcal{H}_{4}$, it immediately follows by (3.14) and the well-known fact that for each $\tau \in \mathcal{H}_{4}$ there are 10 vanishing thetanulls. Finally, since the order of the zeros of $S_{4 i j}(\tau)$ depends on $i, j$ and $\tau$, it follows by (3.14) that $S_{4 i j}(\tau)$ and $\sigma_{i j}(e, \tau)$ have the same divisors in $\mathcal{I}_{4}$. When $e \in \Theta_{s}$ is an even characteristic, $\chi_{68}(\tau)$ has at least a double zero so that $\operatorname{det} S_{4}(\tau)$, and by (3.14), $\operatorname{det} \sigma(e, \tau)$ has a zero of order at least one for all $\tau \in \mathcal{I}_{4}$.

Remark 3.6. In 30] the Klein formula was re-obtained, linking $\chi_{18}$ to the square of the discriminant of plane quartics. The authors also studied possible generalizations to the case $g>3$. In particular, equation (3) of [30 mentions the following "amazing formula" given by Klein in the footnote on p. 462 in [31:

$$
\chi_{68}(\tau)=c^{\prime} \tilde{\Delta}_{4}(C)^{2} T(C)^{8},
$$

with $c^{\prime}$ a constant. This formula relates $\chi_{68}$ to the discriminant $\tilde{\Delta}_{4}(C)$ of the Klein quadric and the tact invariant $T(C)$ of the quadric and of the cubic (see p. 122 of [32]), whose intersection in $\mathbb{P}^{3}$ determines $C$. Note that if $\sum_{i, j=1}^{4} C_{i j} \omega_{i} \omega_{j}=0$ denotes the Klein quadric, then

$$
C_{i j}=\tilde{c} \frac{S_{4 i j}}{T(C)},
$$

with $\tilde{c} \in \mathbb{C}^{\star}$ independent of $\tau$.

The following expression for $\mu_{4,2}$ has been suggested in 33 and in 22 in the context of bosonic string theory. Its proof has been a long-standing problem, and a more rigorous derivation has been provided in [34]. In the present approach it follows immediately.

Theorem 3.7. The $g=4, n=2$ Mumford form is

$$
\mu_{4,2}= \pm \frac{1}{c S_{4 i j}} \frac{\omega_{1} \omega_{1} \wedge \cdots \wedge \widehat{\omega_{i} \omega_{j}} \wedge \cdots \wedge \omega_{4} \omega_{4}}{\left(\omega_{1} \wedge \cdots \wedge \omega_{4}\right)^{13}}
$$

for all $i, j=1, \ldots, 4$, with $c \in \mathbb{C}^{*}$ the constant defined in (3.7).

Proof. Immediate by (2.8) and by (3.7) of Lemma 3.2 . 


\section{REFERENCES}

1. M. Matone and R. Volpato, Higher genus superstring amplitudes from the geometry of moduli space, Nucl. Phys. B $\mathbf{7 3 2}$ (2006), 321-340. MR2192117(2007a:81117)

2. M. Matone and R. Volpato, Superstring measure and non-renormalization of the three-point amplitude, Nucl. Phys. B $\mathbf{8 0 6}$ (2009), 735-747. MR2467587 (2010g:81245)

3. M. Matone and R. Volpato, Getting superstring amplitudes by degenerating Riemann surfaces, Nucl. Phys. B 839 (2010), 21-51. MR2670813 (2011g:32025)

4. M. Matone and R. Volpato, Linear relations among holomorphic quadratic differentials and induced Siegel's metric on $\mathcal{M}_{g}$, J. Math. Phys. 52 (2011), 102305. MR2894590(2012m:14060)

5. M. Matone, Extending the Belavin-Knizhnik "wonderful formula" by the characterization of the Jacobian, JHEP 1210 (2012), 175.

6. J. Fay, Kernel functions, analytic torsion and moduli spaces, Mem. Amer. Math. Soc. 96 (1992). MR 1146600 (93e:58192)

7. J. Fay, Theta Functions on Riemann Surfaces, Springer Lecture Notes in Math. 352, 1973. MR0335789 (49:569)

8. M. Matone and R. Volpato, Determinantal characterization of canonical curves and combinatorial theta identities, Math. Ann. DOI:10.1007/s00208-012-0787-z

9. D. Mumford, Stability of projective varieties, Enseign. Math. 23 (1977), 39-110. MR0450272 (56:8568)

10. A. A. Belavin and V. G. Knizhnik, Algebraic geometry and the geometry of quantum strings, Phys. Lett. B 168 (1986), 201-206. MR830618(87i:81189)

11. L. Bonora, A. Lugo, M. Matone and J. Russo, A global operator formalism on higher genus Riemann surfaces: $b-c$ systems, Commun. Math. Phys. 123 (1989), 329-352. MR 1002042 (91a:81184)

12. J. B. Bost and T. Jolicoeur, A holomorphy property and critical dimension in string theory from an index theorem, Phys. Lett. B 174 (1986), 273-276. MR848539 (88i:81130)

13. J. M. Bismut and D. S. Freed, The analysis of elliptic families. I. Metrics and connections on determinant bundles, Commun. Math. Phys. 106 (1986), 159-176; The analysis of elliptic families. II. Dirac operators, eta invariants, and the holonomy theorem, Commun. Math. Phys. 107 (1986), 103-163. MR853982 (88h:58110a) MR0861886(88h:58110b)

14. L. Alvarez-Gaume, J. B. Bost, G. W. Moore, P. C. Nelson and C. Vafa, Bosonization on higher genus Riemann surfaces, Commun. Math. Phys. 112 (1987), 503-552. MR.908551(88j:81061)

15. D. Ray and I. M. Singer, Analytic torsion for complex manifolds, Ann. Math. 98 (1973), 154-180. MR0383463(52:4344)

16. A. A. Beilinson and Y. I. Manin, The Mumford form and the Polyakov measure in string theory, Commun. Math. Phys. 107 (1986), 359-376. MR866195(88a:32035)

17. E. P. Verlinde and H. L. Verlinde, Chiral bosonization, determinants and the string partition function, Nucl. Phys. B 288 (1987), 357-396. MR896667(88k:81232)

18. G. van der Geer, Siegel modular forms and their applications, The 1-2-3 of modular forms, 181-245, Universitext, Springer, Berlin, 2008. MR2409679 (2010c:11059)

19. M. Matone and R. Volpato, The singular locus of the theta divisor and quadrics through a canonical curve, arXiv:0710.2124 [math.AG].

20. N. I. Shepherd-Barron, Thomae's formulae for non-hyperelliptic curves and spinorial square roots of theta-constants on the moduli space of curves, arXiv:0802.3014 [math.AG].

21. A. A. Belavin, V. Knizhnik, A. Morozov and A. Perelomov, Two- and three-loop amplitudes in the bosonic string theory, Phys. Lett. B 177 (1986), 324-328. MR860363 (88f:81115)

22. A. Morozov, Explicit formulae for one-, two-, three- and four-loop string amplitudes, Phys. Lett. B 184 (1987), 171-176. MR879231(88k:81218a)

23. E. D'Hoker and D. H. Phong, Two-loop superstrings. IV: The cosmological constant and modular forms, Nucl. Phys. B 639 (2002), 129-181. MR.1925297(2004b:81156)

24. T. Ichikawa, On Teichmüller modular forms, Math. Ann. 299 (1994), 731-740. MR.1286895 (95k:14038)

25. T. Ichikawa, Teichmüller modular forms of degree 3, Amer. J. Math. 117 (1995), 1057-1061. MR.1342841 (96e:11067)

26. E. D'Hoker and D. H. Phong, Asyzygies, modular forms, and the superstring measure. II, Nucl. Phys. B $\mathbf{7 1 0}$ (2005), 83-116, MR2126049(2006e:81201) 
27. J.-I. Igusa, Schottky's invariant and quadratic forms, E. B. Christoffel (Aachen/Monschau, 1979), 352-362, Birkhäuser, Basel-Boston, Mass., 1981. MR661078 (83m:10031)

28. J.-I. Igusa, On the irreducibility of Schottky's divisor, J. Fac. Sci. Univ. Tokyo Sect. IA Math. 28 (1981), 531-545. MR656035 (83f:14023)

29. S. Tsuyumine, Thetanullwerte on a moduli space of curves and hyperelliptic loci, Math. Z. 207 (4) (1991), 539-568. MR1119956 (92j:14037)

30. G. Lachaud, C. Ritzenthaler and A. Zykin, Jacobians among abelian threefolds: A formula of Klein and a question of Serre, Math. Res. Lett. 17 (2010), no. 2, 323-333. MR.2644379 (2011f:14074)

31. F. Klein, Zur Theorie der Abel'schen Funktionen, Math. Annalen 36 (1889-90); = Gesammelte mathematische Abhandlungen XCVII, 388-474. MR1510611

32. G. Salmon, Traité de géométrie analytique à trois dimensions. Troisième partie. Ouvrage traduit de l'anglais sur la quatrième édition, Paris, 1892.

33. A. A. Belavin and V. G. Knizhnik, Complex geometry and the theory of quantum strings, Sov. Phys. JETP 64 (1986), 214-228 [Zh. Eksp. Teor. Fiz. 91 (1986), 364-390]. MR888370 (88m:32043)

34. J. M. Guilarte and J. Muñoz Porras, Four-loop vacuum amplitudes for the bosonic string, Proc. Roy. Soc. London Ser. A 451 (1995), 319-329. MR1362484 (97j:81277)

Dipartimento di Fisica "G. Galilei" and Istituto Nazionale di Fisica Nucleare, UniVersità di Padova, Via Marzolo, 8, 35131 Padova, Italy

E-mail address: matone@pd.infn.it

Institut für Theoretische Physik, ETH Zurich, CH-8093 Zürich, Switzerland

Current address: Max-Planck-Institut für Gravitationsphysik, Albert-Einstein-Institute, 14476

Potsdam, Germany

E-mail address: roberto.volpato@aei.mpg.de 